\title{
The structure of the proton, recent results from HERA
}

\author{
loannis Gialas \\ Physics Department, University of Crete \\ P.O. Box 2208, GR-71003 Heraclion \\ and \\ University of Hamburg, II. Institute for Experimental Physics \\ E-mail: gialas@physics.uch.gr
}

ABSTRACT: We report here on some recent results using the ZEUS detector. Using a variety of methods, measurement of $F_{2}$ covers a very wide range $2.0 \times 10^{-6}<x<0.08$ and $0.11<Q^{2}<5000 \mathrm{GeV}^{2}$. The steep rise of the structure function continues down to the lowest measured $x$. Using a new small calorimeter next to the beam pipe (BPC), ZEUS could measure $F_{2}$ down to $Q^{2} \simeq 0.1 \mathrm{GeV}^{2}$. In this way, the transition from the DIS regime to the photoproduction regime can be studied. Fitting the $F_{2}$ data, some conclusions regarding the gluon content of the proton canbe reached. Charm production was measured and was found that it can account for up to $30 \%$ of the total inclusive cross section.

\section{Introduction}

The HERA collider collides $27.5 \mathrm{GeV}$ positrons (or electrons) on $820 \mathrm{GeV}$ protons. The beams intercect in four interaction points. Of these, the Northern interaction point is instrumented with the H1 detector, and the Southern with ZEUS. The analyses covered in this talk used data from positron runs with both the above detectors. The peak HERA luminosity has reached $10^{31} \mathrm{~cm}^{-2} \mathrm{~s}^{-1}$. The following table shows the collected integrated luminosity by ZEUS.

\begin{tabular}{|c|c|}
\hline \multicolumn{2}{c}{ ZEUS evtake } \\
\hline Year & Integr. lumin. $\left(p b^{-1}\right)$ \\
\hline 1994 & 3.0 \\
1995 & 6.3 \\
1996 & 10.7 \\
1997 & 26.6 \\
\hline
\end{tabular}

\section{HERA kinematics}

The interaction between an electron or a positron and a proton in the deep inelastic regime proceeds via the exchange of a photon $\left(\gamma^{*}\right)$ or a $Z^{0}$ in the neutral current events or via the exchange of $W^{ \pm}$in the charged current events. In this picture the boson is exchanged between the lepton and a quark in the proton. The struck quark hadronizes and is detected as concentrated energy deposit (jet). The rest of the proton, hadronizes and deposits energy around the forward (proton direction) beam pipe.

The event is characterized by the four momentum transfer squared, $Q^{2}$, the Bjorken $x$ variable, $x \equiv Q^{2} / 2 p \cdot q$, which in the infinite momentum frame is the fraction of the proton momentum carried by the struck quark, and the inelasticity parameter, $y \equiv p \cdot q / p \cdot k$. In the above definitions, $\mathrm{p}, \mathrm{k}$, and $\mathrm{q}$, are the proton, electron, and exchanged boson four momenta respectively. In terms of the above, the center of mass energy squared is given by $s \equiv(p+k)^{2} \approx Q^{2} / x y$ and the mass of the photon-proton system $W^{2} \equiv$ $(q+k)^{2} \approx Q^{2} / x$.

The $Q^{2}$ can be related to the distances which can be resolved in the proton. With $Q^{2} \leq 0.1 \mathrm{GeV}^{2}$ the photon probes distances of the size of the proton, $d \sim 1 \mathrm{fm}$. For $Q^{2} \simeq 1 \mathrm{GeV}^{2}$, one probes the transition region between hard and soft interactions, $d \sim 0.2 \mathrm{fm}$. As $Q^{2}$ increases, the interaction can see more small $x$ and fewer high $x$ quarks.

The complete kinematic range over which ZEUS 
has measured $F_{2}$ is $0.1<Q^{2}<5000 \mathrm{GeV}^{2}$ and $2 \times 10^{-6}<x<0.5$ and can be seen in figure 11. HERA has extended the accessible kinematic range by two orders of magnitude both in $x$ and $Q^{2}$ compared to previous measurements made by fixed target experiments.

\section{The detectors}

The ZEUS detector is a $4 \pi$ hermetic detector. Going from the interaction point outwards, the succesive elements are the tracking detector, the superconducting solenoid, the Calorimeter, the backing calorimeter and muon chambers.

The asymmetry of the beam energies necessitates the design of the detector to be also asymmetric. The calorimeter is a compensated Depleted Uranium - Scintillator sampling calorimeter. The signal is read out by 12000 photomultipliers WITH 2 PMT's per cell. The resolution, $\sigma / E$, is $18 \% / \sqrt{E}$ for electrons, and $35 \% / \sqrt{E}$ for hadrons. The ratio of electromagnetic to hadronic response is $e / h=1$. The systematic error on the energy scale is about $3 \%$.

A neutral current ZEUS event with $Q^{2}=$ $1600 \mathrm{GeV}^{2}$ can be seen in figure $\overline{2}_{\mathrm{r}}^{-1}$ In the left picture, one sees the $\mathrm{r}-\mathrm{z}$ projection of the detector. The positrons enter from the left and the

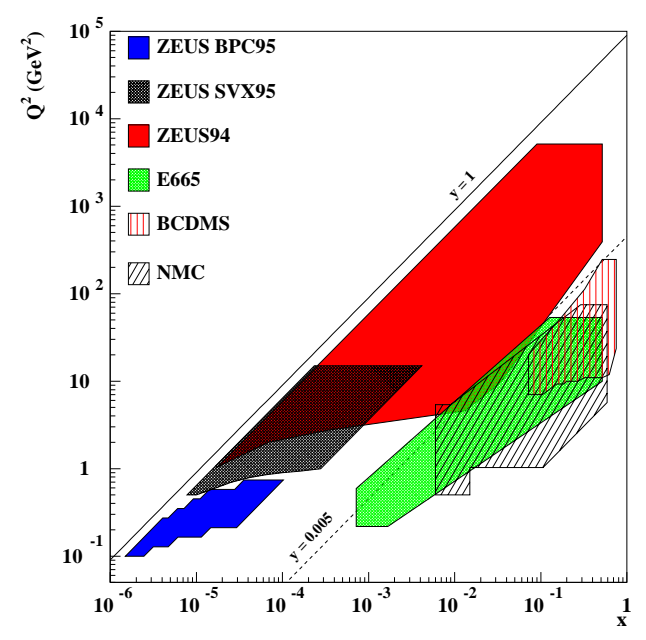

Figure 1: The kinematic range on the $Q^{2}-x$ plane covered by ZEUS. On the same graph the ranges reached by fixed target experiments are also presented. protons from the right. Notice the asymmetry in the depth of the calorimeter in the two directions. One can easily identify the deposit of the positron (up), the current jet (down), and the proton remnant (left). In the right picture, the $\mathrm{x}-\mathrm{y}$ projection of the detector, one can see that the positron and the current jet are emitted back to back, as expected.

\section{Event Reconstruction}

The ZEUS and $\mathrm{H} 1$ detectors offer the capability to measure the final state electron and current jet both in energy and direction. Of the four measured quantities, only two are independent and the rest are redundant. This feature allows for a variety of reconstruction methods with their own advantages and disadvantages. The most widely used methods are the following.

- The electron method. Only the final state lepton energy $E_{e}$ and direction $\theta_{e}$ are used. This method is used by $\mathrm{H} 1$ and in ZEUS for the low $Q^{2}$ analyses. Clearly, it can only be used in the case of neutral current events.

- Double Angle Method The two angles $\theta_{e}$ and $\gamma_{h}$ are used where $\gamma_{h}$ is the angle of the struck quark. Used mainly by ZEUS. Its main advantage is that it is relative insensitive to the electron energy scale.

- Hadronic Activity (Jacquet-Blondel. It does not require final state lepton measurement. It is the only method available to measure charged current events, where a neutrino is emitted in the final state .

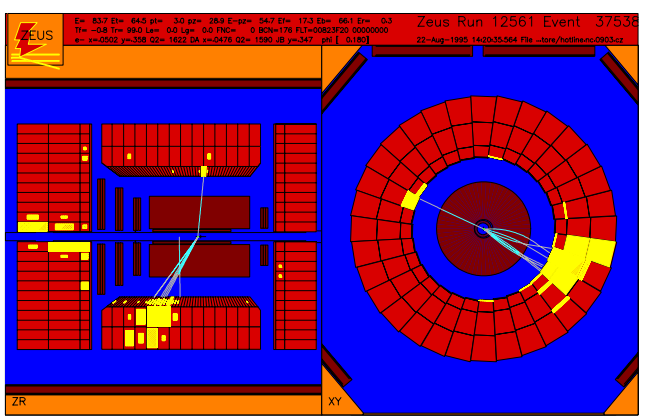

Figure 2: A typical neutral current ZEUS event. 


\section{5. $F_{2}$ measurement}

The neutral current deep inelastic scattering cross section can be parametrized as follows:

$$
\begin{array}{r}
\frac{d^{2} \sigma_{N C}\left(e^{\mp} p\right)}{d x d Q^{2}}=\frac{2 \pi \alpha^{2}}{x Q^{4}}\left[Y_{+} F_{2}\left(x, Q^{2}\right)-y^{2} F_{L}\left(x, Q^{2}\right)\right. \\
\left. \pm Y_{-} x F_{3}\left(x, Q^{2}\right)\right]
\end{array}
$$

where $Y_{ \pm} \equiv 1 \pm(1-y)^{2}, F_{L}$ is the longitudinal structure function, and $F_{3}$ is the parity violating term arising from the $Z^{0}$ exchange.

In the described analysis, the structure function $F_{2}$ is measured. Its meaning is clear within the framework of the parton model. $F_{2}\left(x, Q^{2}\right)=$ $x \sum_{i} e_{i}^{2}\left(q_{i}+\bar{q}_{i}\right)$ is the sum of all the quark and antiquark contributions weighted by the square of their charges. If the proton consisted only of a quark, then $F_{2}$ would be a delta function at $x=1$. If it consisted of three noninteracting quarks, then $F_{2}$ would be a delta function at $x=1 / 3$. In the case of three interacting quarks,

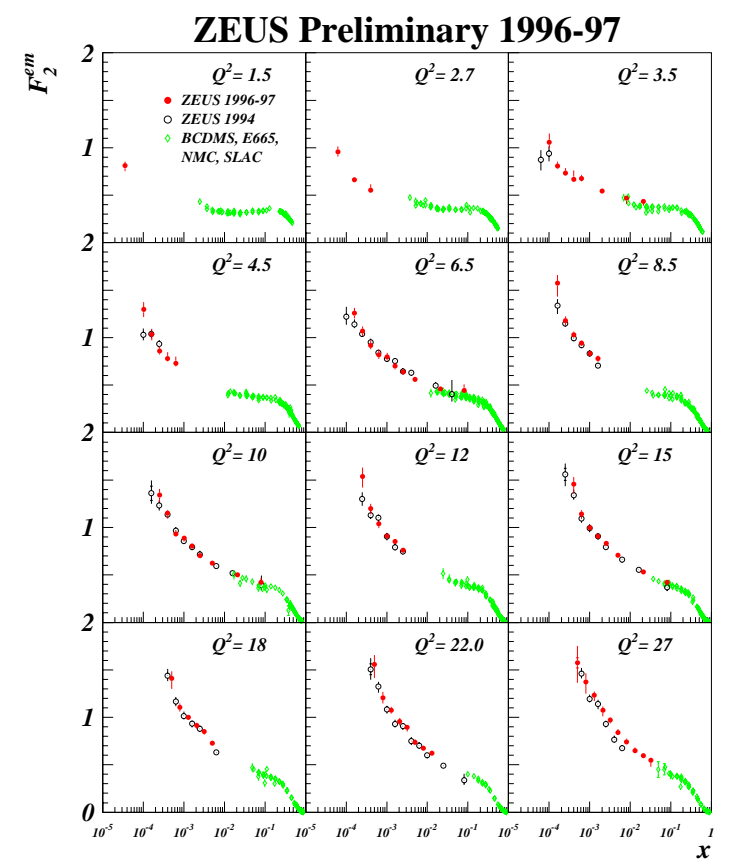

Figure 3: $F_{2}$ measurements from ZEUS against $x$ for different $Q^{2}$ bins with $1.5<Q^{2}<27 \mathrm{GeV}^{2}$. Included measurements are from published ZEUS data (refer) and from new 1994-1997 runs. The data points from fixed target experiments have been included on the same plot. the $F_{2}$ would be peaked at $1 / 3$ with limiting values of 0 as $x \rightarrow 0,1$. Finally, when the possibility of gluon radiation and quark pair production is added, $F_{2}$ is expected to have larger values for $x \rightarrow 0$.

In order to perform the measurement, one has to make some assumptions regarding the size of the contributions due to $F_{3}, F_{L}$ and radiative corrections. $F_{3}$ only becomes appreciable for $Q^{2}$ of the order of $1000 \mathrm{GeV}^{2}$ and higher. The longitudinal structure function, $F_{L}$ becomes appreciable only when $y \rightarrow 1$. All the above contributions are treated as corrections, $\delta_{L}, \delta_{3}, \delta_{r}$ and their magnitude is determined with the use of Monte Carlo.

Then, equation $5 \cdot \bar{b}$ becomes

$$
\begin{aligned}
\frac{d^{2} \sigma_{N C}\left(e^{\mp} p\right)}{d x d Q^{2}} \approx & \frac{2 \pi \alpha^{2}}{x Q^{4}} Y_{+} F_{2}\left(x, Q^{2}\right) \\
& \left(1-\delta_{L}-\delta_{3}\right)\left(1+\delta_{r}\right)(5.2)
\end{aligned}
$$

The next step is to isolate the contribution to $F_{2}$ from the $Z^{0}$ exchange. $F_{2}$ can be written

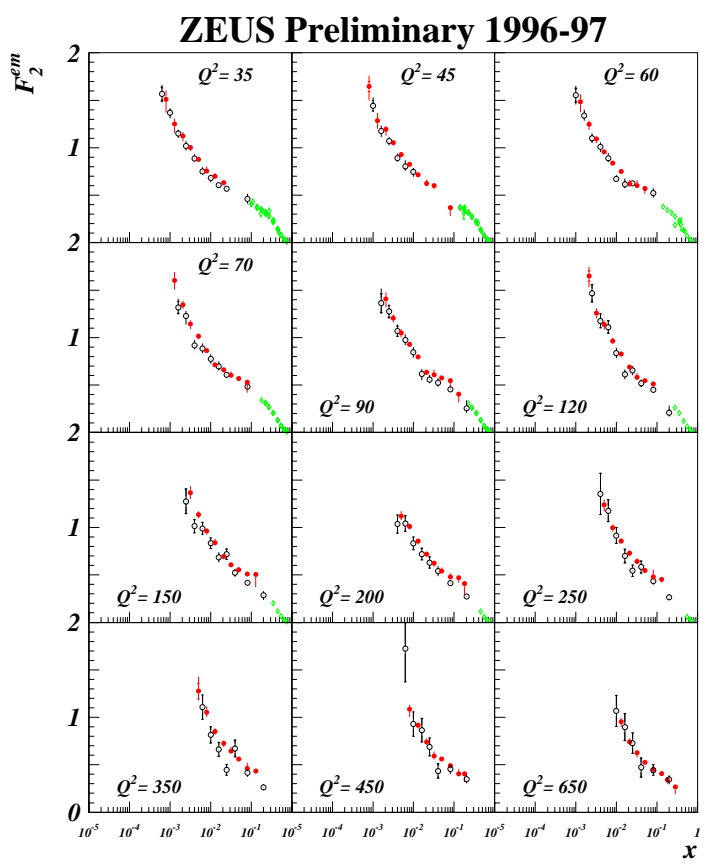

Figure 4: $F_{2}$ measurements from ZEUS against $x$ for different $Q^{2}$ bins with $35<Q^{2}<650 \mathrm{GeV}^{2}$. Included measurements are from published ZEUS data (refer) and from new 1994-1997 runs. The data points from fixed target experiments have been included on the same plot. 
as

$$
F_{2}=F_{2}^{e m}+\left(\frac{Q^{2}}{Q^{2}+M_{Z}^{2}}\right) F_{2}^{i n t}+\left(\frac{Q^{2}}{Q^{2}+M_{Z}^{2}}\right)^{2} F_{2}^{w k}
$$$$
\approx F_{2}^{e m}\left(1+\delta_{Z}\right)
$$

where $F_{2}^{e m}$ is the contribution to $F_{2}$ due to photon exchange, $F_{2}^{w k}$ is the contribution due to $Z^{0}$ exchange, and $F_{2}^{i n t}$ is the contribution due the $\gamma Z^{0}$ interference. $F_{2}^{e m}$ is the dominant contribution in the $Q^{2}$ range under investigation.

Finally the measured $F_{2}$ for positron proton scattering is found from the following formula

$$
\begin{array}{r}
\frac{d^{2} \sigma_{N C}\left(e^{+} p\right)}{d x d Q^{2}} \approx \frac{2 \pi \alpha^{2}}{x Q^{4}} Y_{+} \\
F_{2}^{e m}\left(x, Q^{2}\right)\left(1-\delta_{L}-\delta_{3}\right) \\
\left(1+\delta_{Z}\right)\left(1+\delta_{r}\right)
\end{array}
$$

The ZEUS results on $F_{2}$ versus $x$ can be found in figures $\overline{3}_{1}$ The published ZEUS $F_{2}$ measurements [1] $\left.{ }_{1}^{1}\right]$ based on the 1994 data are plotted along the preliminary measurements from the 1996-1997 data, and

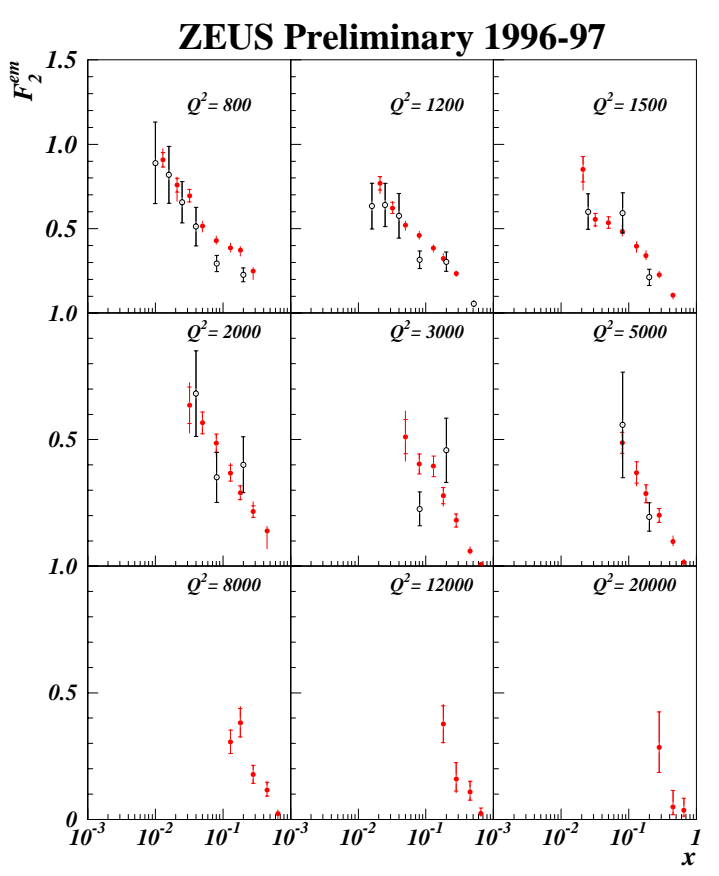

Figure 5: $F_{2}$ measurements from ZEUS against $x$ for different $Q^{2}$ bins with $800<Q^{2}<20000 \mathrm{GeV}^{2}$. Included measurements are from published ZEUS data (refer) and from new 1994-1997 runs. results from fixed target experiments[2]. At low $Q^{2}$ the rise of $F_{2}$ for $x \rightarrow 0$ is measured with improved precision. The coverage in $x$ has has also been extended to higher $x$, yielding considerable overlapwith the fixed target experiments. In the overlap region good agreement has been found. Above $Q^{2} \sim 800 \mathrm{GeV}^{2}$, the statistical error grows typically to $5-15 \%$ and dominates the total error. In figure $\overline{6}_{1}^{1}$ the same data is plotted against $Q^{2}$ in different $x$ bins. The scaling violation is easily observed.

\section{Low $Q^{2}$ region}

The persistence of the strong rise to small values of $Q^{2}$ and the apparent success of the NLO QCD description of the data down to $Q^{2}$ values approaching $1 \mathrm{GeV}^{2}$ raise new questions for our understanding of QCD. HERA allows study of the transition region as $Q^{2} \rightarrow 0$ in which perturbative QCD (pQCD) must break down.

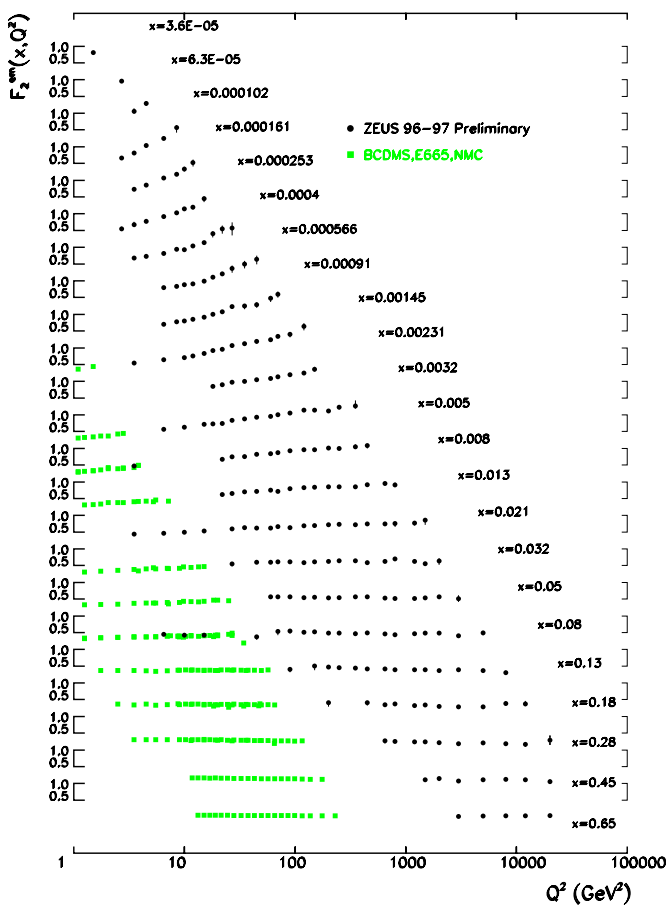

Figure 6: $F_{2}$ measurements from ZEUS against $Q^{2}$ for different $x$ bins. The data points from fixed target experiments have been included on the same plot. 
There is a wealth of data in the photoproduction regime, $Q^{2}=0$. Also, one of the first measurements by HERA was the measurement of photoproduction cross section. A characteristic of the photoproduction cross section is its weak dependence on $\mathrm{W}$.

For $Q^{2}>0$, at low- $x$ and big enough $\mathrm{W}$, the total $\gamma^{*} p$ cross section is related to $F_{2}$ by:

$$
\sigma_{\text {tot }}^{\gamma^{*} p}\left(W^{2}, Q^{2}\right) \equiv \sigma_{T}+\sigma_{L} \approx \frac{4 \pi^{2} \alpha}{Q^{2}} F_{2}\left(x, Q^{2}\right)
$$

In this regime the slope of the cross section is steeper. How does the cross section behaviour change from the photoproduction to the deep inelastic regime?

In order to make it possible to access the low- $Q^{2}$ regime, a small electromagnetic sampling calorimeter (BPC) was installed near the beam pipe, at small positron scattering angles. The data obtained with this detector made it possible to access the $Q^{2}$ region between 0.6 and 0.65 $\mathrm{GeV}^{2}$. The gap between the BPC data and the 1994 ZEUS measurements was bridged by the so called shifted vertex run, whereby the interaction point was shifted away from the ZEUS main rear calorimeter thus extending its small angle coverage for scattered positrons.

In figure $\overline{7}_{1}$ an overview is given of the low$Q^{2}$ region, $Q^{2}<5 \mathrm{GeV}^{2}$, taking ZEUS $F_{2}$ data from the shifted vertex analysis with 1995 data (SVX95), 1995 BPC data (BPC95), and 1994 main calorimeter data (ZEUS94). Using equation 6 , the cross section $\sigma_{\text {tot }}^{\gamma^{*} p}$ was calculated from the low $Q^{2} F_{2}$ data. Also shown are data from H1 SVX95 and measurements of the total cross section for scattering of real photons on protons at fixed target ergies. The two curves shown are: dotted, the

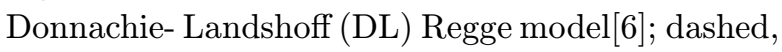
$F_{2}$ calculated from the NLO QCD parton distributions GRV94 [i] the cross section rises slowly with energy $\propto W^{2 \lambda}$ where $\lambda=1-\alpha_{I P} \sim 0.08$ and this behaviour seems to be followed by the data at very low $Q^{2}$ values, although the normalization of the DL model is low compared to the ZEUS BPC95 data. Above $Q^{2}=0.65 \mathrm{GeV}^{2}$, the DL model predicts a shallower rise of the cross section than the data exhibit. For $Q^{2}$ values of around $1 \mathrm{GeV}^{2}$ and above, the GRV94 curves describe the qualitative behaviour of the data, namely, the increasing rise of $\sigma_{\text {tot }}^{\gamma^{*} p}$ with $W^{2}$, as $Q^{2}$ increases. This suggests that the perturbative QCD calculations can account for a significant fraction of the cross section at the larger $Q^{2}$ values.

At a fixed value of $Q^{2}$ and at small $x$ the behaviour of $F_{2}$ can be characterized by $F_{2} \propto x^{-\lambda}$, with $\lambda$ taking rather different values in the Regge and BFKL approaches. The $F_{2}$ behaviour can be quantified by the definition of the observable $\lambda_{e f f} \equiv\left\langle\frac{d \ln F_{2}}{\operatorname{dln}(1 / x)}\right\rangle$. Refering to figure $\lambda_{1}^{1} \lambda_{\text {eff }}$ was calculated from horizontal slices of ZEUS $F_{2}$ data between the $y=1$ HERA kinematic limit and a fixed cut of $x<0.01$ below which the contribution from valence quarks to $F_{2}$ becomes negligible. The figure shows that $x$ and $Q^{2}$ are correlated and in a given $Q^{2}$ bin $\langle x\rangle$ is calculated from the mean value of $\ln (1 / x)$ weighted by the statistical errors of the corresponding $F_{2}$ values in that bin.

Figure $\bar{s}_{i}$ shows the measured values of $\lambda_{\text {eff }}$ as a function of $Q^{2}$. From the Regge approach one would expect $\lambda_{\text {eff }} \approx 0.1$ and independent of $Q^{2}$. The linked points labelled DL are calculated from the Donnachie-Landshoff fit and are some-

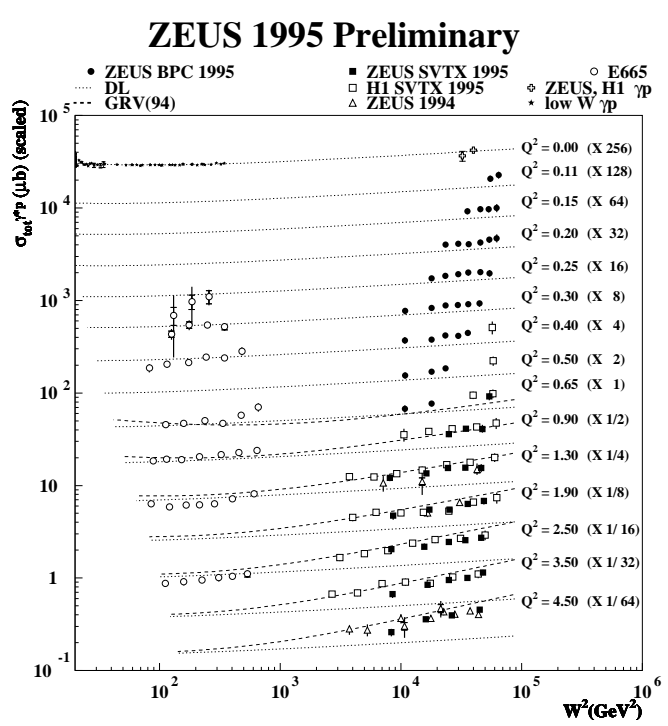

Figure 7: The $\sigma_{t o t}\left(\gamma^{*} p\right)$ is plotted versus $W^{2}$ for different $Q^{2}$ bins. The set of data points on top corresponds to photoproduction, $Q^{2}=0$ 
what below the data. For $Q^{2}>1 \mathrm{GeV}^{2}, \lambda_{\text {eff }}$ increases slowly to around 0.3 at $Q^{2} \approx 40 \mathrm{GeV}^{2}$. Qualitatively the tendency of $\lambda_{\text {eff }}$ to increase with $Q^{2}$ is described by a number of pQCD approaches [3]. The linked points labelled GRV94 are calculated from the NLO QCD GRV94 fit. Although the GRV94 prediction follows the trend of the data it tends to lie above the data, particularly in the $Q^{2}$ range $3-20 \mathrm{GeV}^{2}$.

Within the framework of pQCD, at small $x$ the behaviour of $F_{2}$ is largely determined by the behaviour of the sea quarks, whereas the $d F_{2} / d \ln Q^{2}$ is determined by the convolution of the splitting function and the gluon density. In order to study the scaling violations of $F_{2}$ in more detail the logarithmic slope $d F_{2} / d \ln Q^{2}$ is derived from the data by fitting $F_{2}=a+b \ln Q^{2}$ in bins of fixed $x$. The results are shown in figure $\overline{p_{r}^{1}}$ For values of $x$ down to $3 \times 10^{-4}$, the slopes are increasing as $x$ decreases. At lower values of $x$ and $Q^{2}$, the slope decreases. If $d F_{2} / d \ln Q^{2}$ is plotted for fixed target data, the 'turn over' is not seen, but of course the data is at larger values of $x$. Comparing the rapid increase in $F_{2}$ at small $x$ with

\section{ZEUS 1995 Preliminary}
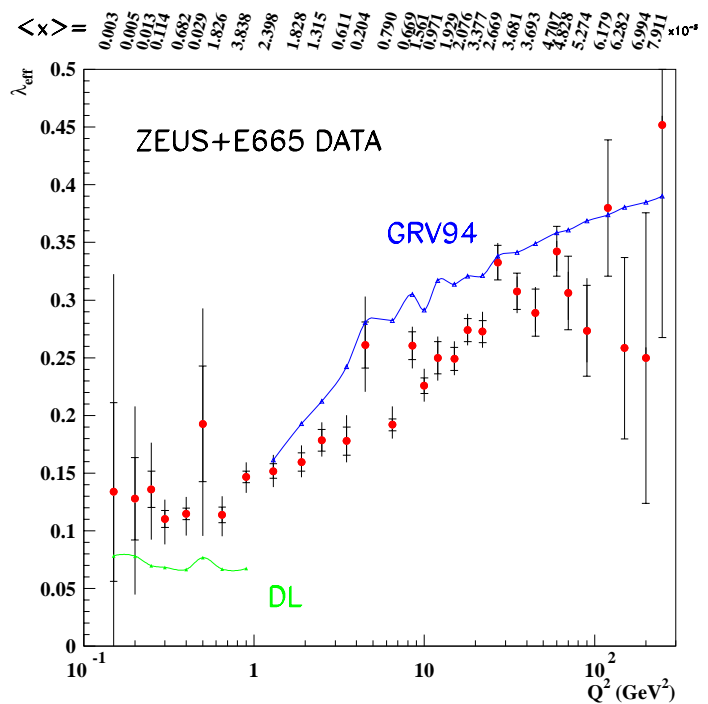

Figure 8: The quantity $d F_{2} / d \ln x$ is plotted against $Q^{2}$. The drawn line on the low- $Q^{2}$ side of the plot is the predictions from soft Pomeron exchange based model. The line on the high- $Q^{2}$ side of the plot is a prediction from a pQCD based model. the behaviour of the $d F_{2} / d \ln Q^{2}$, one is tempted to the naive conclusion that the underlying behaviour of the sea quark and gluon momentum distributions must be different at small $x$, with the sea dominant and the gluon tending to zero. The linked points are again from the DL Regge fit and the GRV94 parametrization.

\section{Extraction of gluon density from ZEUS data}

In pQCD the scaling violations of the $F_{2}$ structure function are caused by gluon bremsstrahlung from quarks and quark pair creation from gluons. In the low $x$ domain accessible at HERA the latter process dominates the scaling violations. A QCD analysis of $F_{2}$ structure functions measured at HERA therefore allows one to extract the gluon momentum density in the proton down to low values of $x$. Figure 10 distribution as a function of $x$ for $Q^{2}$ at 1,7 , and $20 \mathrm{GeV}^{2}$.

The QCD evolution equations are written in terms of quark flavour singlet and non-singlet

\section{ZEUS 1995 Preliminary}
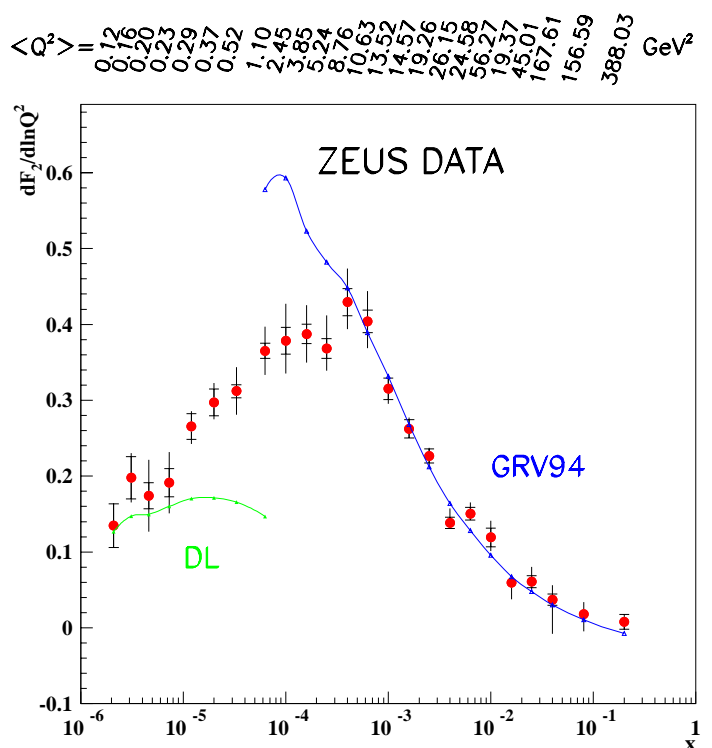

Figure 9: The quantity $d F_{2} / d \ln Q^{2}$ is plotted against $x$. The drawn line on the low- $x$ side of the plot is the predictions from soft Pomeron exchange based model. The line on the high- $x$ side of the plot is a prediction from a pQCD based model. 
and gluon momentum distributions. The quark nonsinglet distribution is independent of the gluon. The quark singlet distribution is defined as the sum over all quark and anti-quark distributions

$$
\Sigma=\sum_{i=1}^{f}\left[q_{i}(x)+\bar{q}_{i}(x)\right]
$$

and its evolution in $Q^{2}$ is coupled to that of the gluon distribution. At small values of $x, x \Sigma$ is dominated by the contribution from the $q \bar{q}$ sea $x S$.

The three plots of figure $\overline{1}_{1}^{1}$, show the distributions for $x \Sigma$ and $x g$ as functions of $x$ for $Q^{2}$ at 1, 7, and $20 \mathrm{GeV}^{2}$. The error bands shown correspond to the quadratic sum of all error sources. It can be seen that even at the smallest $Q^{2} x \Sigma$ is rising at small $x$ whereas the gluon distribution has become almost flat. These results give support to the conclusion that the sea distribution dominates at low $x$ and $Q^{2}$. At $Q^{2}=1 G e V^{2}$ the gluon distribution is poorly determined and can, within errors, be negative at low $x$. In the simplest form of the parton model this would clearly be unphysical. However, a negative gluon dis-

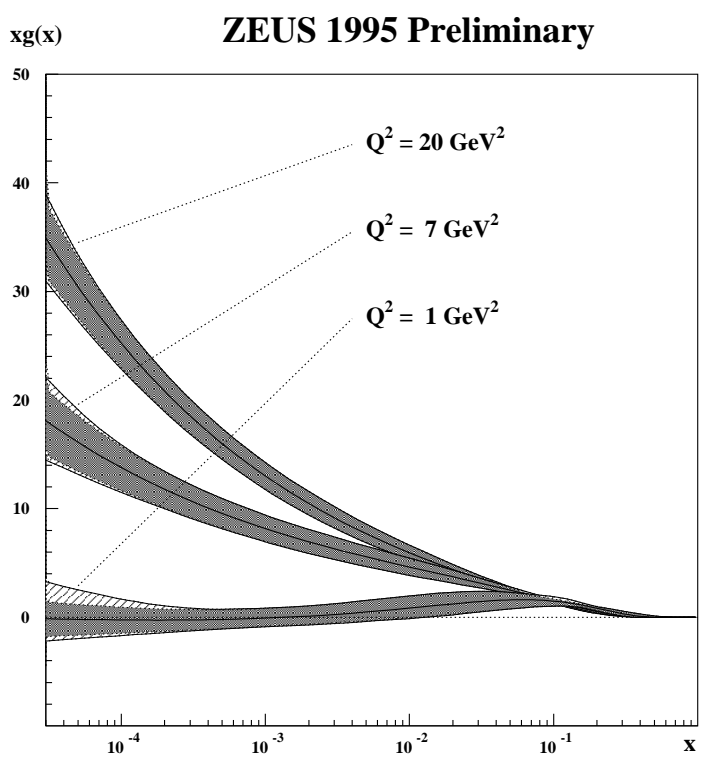

Figure 10: The gluon structure function $x g(x)$ is plotted as a function of $x$ for three different values of $Q^{2}$. The inner shaded bands show the HERA standard errors. The outer hatched bands indicate the quadratic sum of the 'HERA standard' and the parametrization errors. tribution is not necessarily in contradiction with perturbative NLO QCD as long as cross sections or structure functions calculated from the parton distributions are positive for all $x$ and $Q^{2}$ in the fitted kinematic domain.

\section{Charm structure function in DIS}

Measurements of $D^{* \pm}$ production in deep inelastic scattering were made using the data sets 1996 and 1997 with a total integrated luminosity of $37 p b^{-1}$, and the decay channel $D^{* \pm} \rightarrow\left(D^{0} \rightarrow\right.$ $\left.K^{-} \pi^{+}\right) \pi^{+}$(+c.c.). The cross section for inclusive $D^{* \pm}$ production with $1<Q^{2}<600 \mathrm{GeV}^{2}$ and $0.02<y<0.7$ is $8.55 \pm 0.31$ (stat) ${ }_{-0.50}^{+0.30}$ (syst) $n b$ in the kinematic region $1.5<p_{T}\left(D^{* \pm}\right)<15 \mathrm{GeV}$ and $\left|\eta\left(D^{* \pm}\right)\right|<1.5$. The cross section is extrapolated to the full kinematic range in $p_{T}\left(D^{* \pm}\right)$ and $\eta\left(D^{* \pm}\right)$ in order to determine the open charm contribution, $F_{2}^{c \bar{c}}\left(x, Q^{2}\right)$, to the proton structure function, $F_{2}$, at $Q^{2}$ between $1.8 \mathrm{GeV}^{2}$ and 130 $\mathrm{GeV}^{2}$ and Bjorken $x$ between $5 \times 10^{-5}$ and $2 \times$ $10^{-2}$.

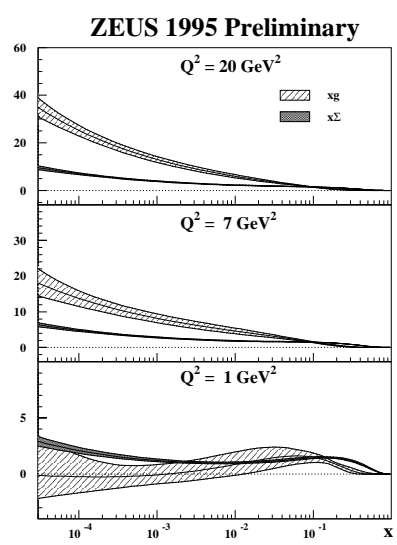

Figure 11: The quark singlet momentum distribution, $x \Sigma$ (shaded), and the gluon momentum distribution, $x g(x)$ (hatched), as functions of $x$ at fixed values of $Q^{2}=1,7$, and $20 \mathrm{GeV}^{2}$. The error bands correspond to the quadratic sum of all error sources considered for each parton density. 
The structure function $F_{2}^{c \bar{c}}\left(x, Q^{2}\right)$ was calculated from the inclusive cross section using the formula

$\frac{d^{2} \sigma_{c \bar{c}}\left(x, Q^{2}\right)}{d x d Q^{2}}=\frac{2 \pi \alpha^{2}}{x Q^{4}}\left[\left[1+(1-y)^{2}\right] F_{2}^{c \bar{c}}\left(x, Q^{2}\right)\right]$

Figure 112 shows the results in the various $Q^{2}$ bins as a function of $x$. The results of the present analysis are shown as solid circles. With the present sample the errors are dominated by statistics. The results are in agreement with the NLO QCD calculation with the GRV94 HO pdf's as input.The shaded band of theoretical predictions corresponds to variation of the $m_{c}$ between 1.2 and $1.6 \mathrm{GeV}$. On the same plot, the preliminary ZEUS result on $F_{2}^{c \bar{c}}$ from an analysis of semileptonic charm decays in the 1995 data sample is shown. The two independent results are in good agreement.

Figure ${ }^{1} \overline{2}_{1}^{1}$ shows $F_{2}^{c \bar{c}}$ at various constant $x$ values as functions of $Q^{2}$. Although the number of points is small, large scaling violations of the structure function can be observed.

Figure 114, shows the ratio of $F_{2}^{c \bar{c}}$ over the proton structure function $F_{2}$ as a function of $x$. For determining the ratio for the data points the value of $F_{2}$ was taken from the ZEUS NLO QCD fit at the same $Q^{2}, x$ for which $F_{2}^{c \bar{c}}$ was quoted.

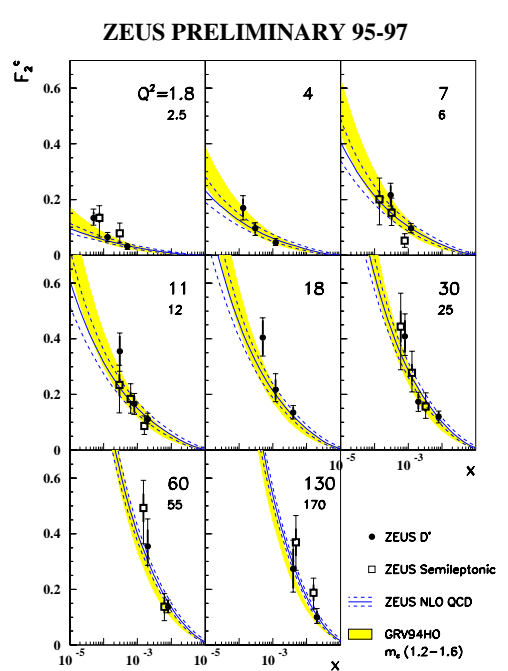

Figure 12: $F_{2}^{\text {charm }}$ versus $x$ for different $Q^{2}$ bins. The shaded band indicates the prediction by GRV94HO.
The error on $F_{2}$ was neglected. The charm contribution to $F_{2}$ is rising steeply with decreasing $x$ at low $Q^{2}$ and becomes flatter as $Q^{2}$ increases. In the measured $x$ region, $F_{2}^{c \bar{c}}$ can account for $20-25 \%$ of $F_{2}$ on average, a contribution which rises up to $30 \%$ at high $Q^{2}$.

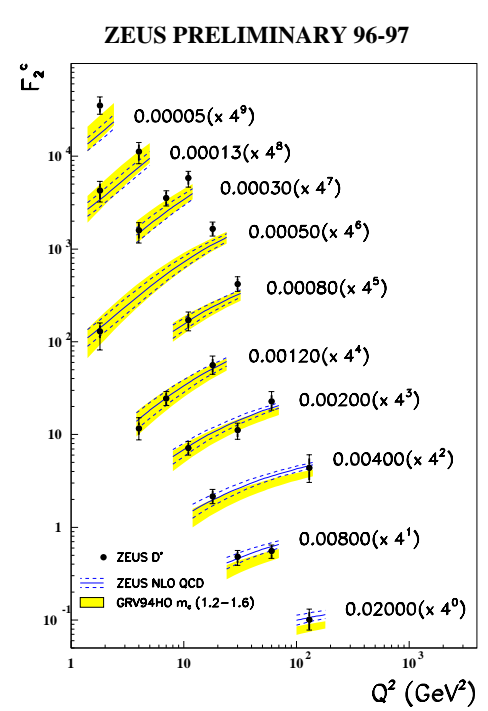

Figure 13: $F_{2}^{\text {charm }}$ versus $Q^{2}$ for different $x$ bins. The shaded band indicates the prediction by GRV94HO. 
9. Conclusion

[4] D.O. Caldwell et al., Phhys. Rev. Lett. 40 1978$)_{1}^{\prime}$ '-20-12

HERA has opened up a new regime in the exploS.I. Alekhin et al., CERN-HERA 87-01(1987).

ration of the proton. The structure function $F_{2}$ is seen to increase in all $Q^{2}$ when $x$ is decreasing. The Regge motivated models describe the data for $Q^{2} \leq .065 G e V_{-}^{2}$ while_pQCD_motivated models can describe well the data for $Q^{2}>0.9 \mathrm{GeV}^{2}$. It is seen that at low- $x$ the sea quark densities which relate to $F_{2}$ increase while the gluon den; sity flattēens off. The structure function ${ }^{2}{ }_{2}^{c c}$ was measured and it was found that it can account for up to $30 \%$ of the positron-proton cross section.

[5] ZEUS Collaboration, M. Derrick et al., 'Z̈.' Physik C 63 (1994) 391;

H1 Collaboration, A. Aid et al., 'Z. Physik C.69! $(\overline{1} 995) \overline{2} \overline{7}$

[6] A. Donnachie and P. Landshoff, 'ZZ. Physik C61' (1994) 139

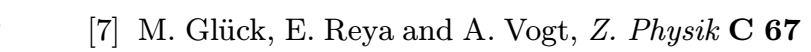
$(1995)-433$

\section{References}

[1] ZEUS collaboration, M. Derrick et al., 'Z- $\bar{P} \bar{P} \bar{y}$ sik' -

- - - ZEUS collaboration, M. Derrick et al., 'Z'- Physik' - - $699(1996) 607$.

[2] BCDMS Collaboration, Benvenuti et al., Pי Phys.' - - - Lett. B 223 (1989) 485

NMC Collaboration, M. Arneodo et al., 'Nucl.' Phys. B $\mathbf{4 8 3}(1997) 3_{3}$

- L. W. Whitlow et al., Phys. Lett. B.282 (1992)! - - - 475

E665 Collaboration, M. R. Adams et al., FERMILAB-PUB-95-396-E.

[3] H. Navelet et al., IPhys. Lett. B $\mathbf{3} 85(1996) 357$.

ZEUS PRELIMINARY 96-97

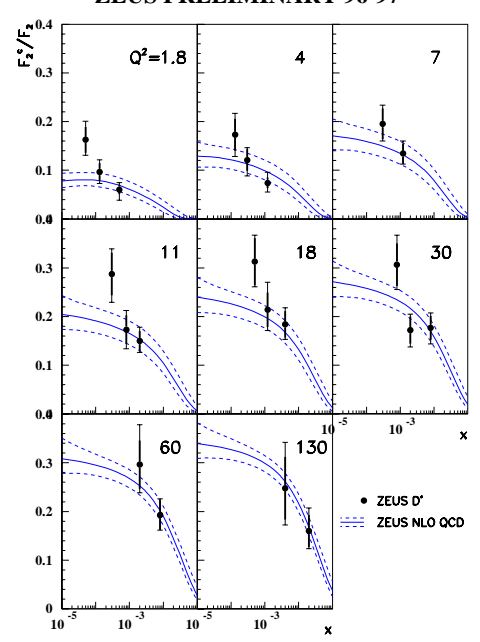

Figure 14: The ratio $F_{2}^{\text {charm }}$ over $F_{2}^{D I S}$ is plotted versus $x$ for different $Q^{2}$ bins. 